\title{
A Preliminary Phase-2 Study with very High- Dose of Melatonin (1000 mg/day) in Untreatable Advanced Cancer Patients Already Progression on Previous Palliative Therapy with High-Dose Melatonin
}

\author{
Paolo Lissoni ${ }^{1 \star}$, Giorgio Porro ${ }^{1}$, Carla Galli ${ }^{2}$, Rosalia Codogni ${ }^{2}$, Nicoletta Merli ${ }^{3}$, Giuseppe Di Fede ${ }^{1}$ \\ ${ }^{1}$ Institute of Biological Medicine, Milan, Italy \\ ${ }^{2}$ Studio Medico Codogni,Verona, Italy \\ ${ }^{3}$ Studio il Faro,Melegnano, Milan, Italy
}

*Corresponding Author: Paolo Lissoni, Institute of Biological Medicine, Milan,Italy. E-mail: paolo.lissoni@, gmx.com

\begin{abstract}
It is known since more than 50 years that the pineal gland plays a fundamental role in the natural biological resistance against cancer onset and growth. The anticancer role of the pineal gland is due to the production of several antitumor molecules, the most known of them is the indole hormone melatonin (MLT). Unfortunately, although there are a lot of experimental evidences that MLT is completely non-toxic, no oncologist seem to be interested to the clinical use of MLT in the treatment of human neoplasms, at least from a palliative point of view. However, a few number of clinical studies of MLT in the complementary treatment of cancer has demonstrated that the anticancer activity of MLT is a dose-dependent phenomenon. On this basis, a preliminary study with a very high dose of MLT, consisting of $1000 \mathrm{mg} /$ once day in the evening, was carried out in untreatable cancer patients, who failed to respond to the conventional anticancer therapies, and for whom the only available treatment was the palliative therapy. The treatment was well tolerated, and a disease control was achieved in 54\% patients. These preliminary results would justify further clinical studies to generate a new interpretation of cancer control, consisting of the pharmacological reproduction of the same mechanisms responsible for the natural resistance against cancer development.
\end{abstract}

Keywords

Cancer, melatonin, palliative therapy, pineal gland

c) (i) The Author(s) 2020. Open Access This article is licensed under a Creative Commons Attribution 4.0 International License (https://creativecommons.org/licenses/by/4.0/), which permits unrestricted use, sharing, adaptation, distribution and reproduction in any medium or format, for any purpose, even commercially, as long as you give appropriate credit to the original author(s) and the source, provide a link to the Creative Commons license, and indicate if changes were made. 


\section{INTRODUCTION}

In normal conditions, the human body has a natural anticancer resistance, which is due to the secretion of molecules provided by immunostimulating and anti-proliferative activity, the most known of them is the pineal indole hormone melatonin (MLT) ${ }^{[1]}$, even though it is not the only pineal hormone provided by anticancer action ${ }^{[2]}$. Moreover, it has been demonstrated that cancer progression is characterized by a progressive decline in MLT production ${ }^{[1]}$. Then, the diminished production of MLT would constitute the main cancer-related endocrine deficiency ${ }^{[1]}$. MLT plays an antitumor action by exerting both antiproliferative activity and stimulatory effects on the anticancer immunity ${ }^{[1]}$. The antiproliferative cytotoxic activity of MLT on tumor cells is due to the induction of the apoptosis of cancer cells and the inhibition of tumor growth factor receptor expression and activation. On the other hand, the anticancer immunostimulatory action of MLT is depending on several complex mechanisms, which include a direct stimulation of Th1-dependent secretion of IL- $2^{[3]}$, which is the main anti-cancer cytokine in humans ${ }^{[4]}$, an inhibition of the release of several inflammatory pro-tumoral cytokines, namely TNF-alpha, IL$6^{[5]}$ and IL-1 $17^{[6]}$, as well as the secretion of anti-inflammatory immunosuppressive pro-tumoral cytokines, namely TGF-beta ${ }^{[7]}$, and stimulation of dendritic cell production of IL-12 ${ }^{[8]}$, which is the main stimulating factor for Th1 differentiation and IL-2 secretion ${ }^{[9]}$. Finally, even though most Oncologists do not know its function, at present MLT is the only existing molecule in our planet, which is able to counteract the overall six major mechanisms responsible for tumor onset and progression ${ }^{[1,3,10,11,12,13,14]}$, including: 1) reversal of stress-induced promotion of tumor development ${ }^{[11]}$; 2) inhibition of spontaneous and carcinogen-induced cancer cell transformation ${ }^{[12]}$; 3 ) contrast of intercellular junction damage ${ }^{[13]}$; 4) inhibition of the neo-angiogenesis following changes in the intercellular matrix $\left.{ }^{[14]} ; 5\right)$ cancer cell proliferation and release of immunosuppressive factors ${ }^{[15]}$; 6 ) inhibition of tumor expression of FAS-ligand, which makes tumor cells as resistant to the cytotoxic action of cytotoxic T lymphocytes ${ }^{[16]}$. The first study of MLT in the treatment of advanced cancer patients, who failed to respond to the conventional anticancer therapies, began in 1989 by using MLT at $20 \mathrm{mg} /$ once day in the evening ${ }^{[17]}$, according to the experimental studies of Bartsch et $a l^{[18]}$, who demonstrated that MLT may exert in vivo an anticancer action only at pharmacological doses and when it is administered during the dark period of the day. Successive studies also demonstrated the possibility to associate MLT with either cancer chemotherapy ${ }^{[19]}$, or IL-2 cancer immunotherapy ${ }^{[20]}$ to enhance their efficacy and reduce their toxicity. Finally, further studies demonstrated the dose-dependency of MLT antitumor action on cancer patients ${ }^{[21,22]}$. On these bases, a study was planned to evaluate the efficacy and tolerability of very high-dose MLT in advanced cancer patients, for whom no other standard antitumor therapy was available, and therefore were eligible for the only best supportive care.

\section{PATIENTS AND METHODS}

The study included 14 consecutive advanced cancer patients, with a life expectancy less than 6 months, who were suitable for the only palliative therapy (M/F: 6/8; median age 65 years; range 33-81). According to ECOG criteria, their median performance status was 2 (range 1-3). Eligibility criteria were as follows: histologically proven solid tumor, measurable lesions, advanced disease, lack of response to previous standard anticancer treatments, and progression even with a previous palliative therapy with MLT at $100 \mathrm{mg} / \mathrm{day}$. Tumor histotypes were, as follows: brain glioblastoma: 5; pancreatic adenocarcinoma: 3 ; breast cancer:2; uterine cervix carcinoma: 1 ; ovarian carcinoma:1; small cell lung cancer: 1 ; malignant astrocytoma: 1 . Distant organ metastases were present in 8/14 patients, and dominant metastasis sites were, as follows: lung:2; liver: 4; brain: 2 . The clinical experimental protocols were approved by the local Ethic Committee of our Institute. Then, it was explained to each patent, and written consents were obtained. MLT was given orally in the night, generally 30 minutes before sleeping. Starting at a dose of $100 \mathrm{mg} / \mathrm{day}$, we reached the maximal dosage of $1000 \mathrm{mg} /$ day within one week, by increasing the dose of $200 \mathrm{mg}$ every next day. Patients continued the treatment at the same MLT dose of $1000 \mathrm{mg} /$ day every day without interruption until disease progression. The dose of $1000 \mathrm{mg} /$ day was decided on the basis of the maximal dose previously described in the literature in healthy subjects ${ }^{[23]}$. Radiological examinations, including CT scan, 
NMR and PET, were repeated at 3-month intervals until disease progression. The clinical response was evaluated according to WHO criteria, and patients were considered as evaluable when they were treated for at least one month. Data were statistically analyzed by the chi-square test and the Student's t test, as appropriate.

\section{RESULTS}

Evaluable patients were 13/14, while the last patient with breast cancer rapidly progressed within the first two weeks of treatment. No objective tumor regression was achieved. However, a stable disease (SD) was obtained in 7/13 (54\%) evaluable patients (glioblastoma: 2; pancreatic adenocarcinoma: 2; breast cancer: 1; cervix carcinoma: 1; malignant astrocytoma: 1). The clinical characteristics of the 13 evaluable patients and their clinical response were reported in Table 1. The median duration of response was 5 months (range 3-11+ months). The percentage of SD achieved by patients, who had no progressive disease (PD) on the previous MLT therapy at $100 \mathrm{mg} / \mathrm{day}$, was significantly higher than that obtained in patients who had a PD $(5 / 8$ vs $1 / 6, P<0.05)$. Moreover, lymphocyteto-monocyte ratio (LMR) observed after 3 months of MLT therapy increased in patients with SD, whereas it decreased or remained unchanged in patients with PD. Then, LMR mean values observed after 3 months of therapy in patients with SD was significantly higher than that found in patients, who had a PD $(2.6+/-0.3 v s 1.4$ +/- O.2, X +/- SE, $P<0.05$ ). MLT therapy was well tolerated by all patients, and the only little side-effect was sleepiness in $1 / 13(8 \%)$, while no alteration in the common laboratory examinations was found.

Table 1. Clinical characteristics and clinical response of $\mathbf{1 3}$ evaluable advanced cancer patients eligible for the only supportive care and treated by high-dose melatonin.

\begin{tabular}{|c|c|c|c|c|c|c|}
\hline N & Sex & Age & Tumor histotype & Metastasis sites & $\begin{array}{c}\text { Clinical response } \\
\text { (WHO) }\end{array}$ & $\begin{array}{c}\text { Response } \\
\text { duration (months) }\end{array}$ \\
\hline 1 & F & 33 & Breast cancer & Brain, liver, bone & SD & 5 \\
\hline 2 & F & 51 & Pancreatic adenocarcinoma & Liver & PD & - \\
\hline 3 & M & 74 & Glioblastoma & - & PD & - \\
\hline 4 & F & 81 & Pancreatic adenocarcinoma & Liver & SD & $10+$ \\
\hline 5 & F & 52 & Glioblastoma & - & PD & - \\
\hline 6 & M & 72 & Small cell lung cancer & Lung & PD & - \\
\hline 7 & F & 71 & Glioblastoma & - & PD & - \\
\hline 8 & F & 58 & Glioblastoma & - & SD & $10+$ \\
\hline 9 & F & 72 & Triple negative breast cancer & Brain, lung & PD & - \\
\hline 10 & M & 61 & Pancreatic adenocarcinoma & Liver & SD & 3 \\
\hline 11 & F & 69 & Glioblastoma & - & SD & 5 \\
\hline 12 & F & 65 & Uterine cervix carcinoma & Lung & SD & 4 \\
\hline 13 & M & 36 & Malignant astrocytoma & - & SD & $11+$ \\
\hline *SD: stable disease; PD: progressive disease &
\end{tabular}

\section{DISCUSSION}

The results of this preliminary study show that very high-dose MLT may exert antiproliferative action in patients for whom no other standard anticancer therapy was available, and with poor clinical conditions and they are eligible for the only supportive care. Moreover, this study seems to suggest that disease control under veryhigh dose MLT was more easily achieved in patients, who had already obtained a disease control on a previous therapy with high-dose MLT, by confirming the dose-dependency of the antitumor activity of MLT. Moreover, this study shows that MLT-induced stabilization of tumor growth is associated with an improvement in the immune status of patients, as shown by the increase in LMR. Despite the previously described absolute lack of MLT toxicity in healthy subjects also at a very high dosage, corresponding to $1000 \mathrm{mg} /$ day ${ }^{[23]}$, it is surprising 
that more than 40 years were required before administering the same high dose in untreatable cancer patients, at least to improve their quality of life. Moreover, this preliminary study may also suggest that very high-dose MLT has no toxicity also in cancer patients with a poor clinical status, as well as in the healthy subjects ${ }^{[23]}$. Then, with respect to the previous results reported in the healthy subjects, who received high-dose MLT for no more than 30 days $^{[23]}$, this study shows that MLT at $1000 \mathrm{mg}$ /day may be administered for a longer period of time until more than 11 months without any toxicity, despite the poor clinical status of patients. Therefore, in the successive clinical studies performed in untreatable advanced cancer patients, MLT could be directly administered at 1000 $\mathrm{mg} / \mathrm{day}$, without any risk of toxicity. Further studies with placebo control will be required to better confirm the clinical efficacy of high-dose MLT. Moreover, since the in vivo action of MLT on tumor growth could depend not only on a direct action on tumor cells, but also on the activation of an effective anticancer immune response ${ }^{[3,5,6]}$, successive researches will be required to better establish whether MLT anticancer efficacy may be mediated by the immunobiological response of patients, or namely through a direct antitumor antiproliferative activity.Finally, to better define the in vivo antitumor efficacy of MLT in relation to the different types of tumors, further studies will be required in a greater number of patients affected by the same cancer histotype.

\section{REFERENCES}

[1] Bartsch C, Bartsch H. Melatonin in cancer patients and in tumor-bearing animals. Adv Exp Med Biol 467: 247-264, 1999.

[2] Sze SF, Ng TB, Liu WK. Antiproliferative effect of pineal indoles on cultured tumor cell lines[J]. J Pineal Res, 1993, 14(1): 27-33.

[3] Maestroni GJM. The immunoneuroendocrine role of melatonin[J]. J Pineal Res, 1993, 14(1): 1-10.

[4] EA Grimm, RJ Robb, JA Roth, LM Neckers, LB Lachman, DJ Wilson, SA Rosenberg. Lymphokineactivated killer cell phenomenon.III. Evidence that IL-2 is sufficient for direct activation of peripheral blood lymphocytes into lymphokine-activated killer cells[J]. J Exp Med, 1983,158(4): 1356-1361.

[5] Guerrero JM, Reiter RJ. Melatonin-immune system relationships[J]. Curr Topics Med Chem,2002,2(2): 167179.

[6] Kuklina EM, Glebezdina NS, Nekrasova V. Role of melatonin in the regulation of differentiation of T cells producing interleukin-17 (Th17)[J]. Bull ExpBiol Med,2016, 160(5): 656-658.

[7] Wang YR, Hong RT, Xie YY, Xu JM. Melatonin ameliorates liver fibrosis induced by carbon tetrachloride in rats via inhibiting TGF-beta 1/Smad signalling pathway[J]. Curr Med Sci,2018, 38(2): 236-244.

[8] Lissoni P, Pittalis S, Rovelli F, Vigoré L, Roselli MG, Brivio F. Interleukin-2, melatonin and interleukin-12 as a possible neuroimmune combination in the biotherapy of cancer[J]. J Biol Regul Homeost Agents 1995,9(2): 63-66.

[9] Banks RE, Patel PM, Selby PJ. Interleukin 12: a new clinical player in cytokine therapy[J]. Br J Cancer 1995,71(4): 655-659.

[10] Regelson W, Pierpaoli W. Melatonin: a rediscovered antitumor hormone? Its Relation to Surface Receptors; Sex Steroid Metabolism, Immunologic Response, and Chronobiologic Factors in Tumor Growth and Therapy[J]. Cancer Invest, 1987,5(4): 379-85.

[11] Buswell RS. The pineal and neoplasia. Lancet 1975, 305(7897): 34-35.

[12] Reiter RJ. Mechanisms of cancer inhibition by melatonin[J]. J Pineal Res, 2004, 37(3): 213-214.

[13] Vinken M, Henkens T, De Rop F, Fraczek J, Vanhaecke T, Rogiers V. Biology and pathology of gap junctional channel in hepatocytes[J]. Hepatology,2008, 47(3): 1077-1088.

[14] Park SY, Jang WJ, Yi EY, Jang JY, Jung Y, Jeong JW, Kim YJ. Melatonin suppresses tumor angiogenesis by inhibiting HIF- 1 alpha stabilization under hypoxia[J]. J Pineal Res 2010,48(2): 178-184.

[15] Valverde P, Benedito E, Solano F, Oaknin S, Lozano JA, Garcia-Borron JC. Melatonin antagonizes alphamelanocyte-stimulating hormone enhancement of melanogenesis in mouse melanoma cells by blocking the 
hormone-induced accumulation of the c locus tyrosinase[J]. Eur J Biochem,1995 232(1): 257-263.

[16] Pedrosa AM, Weinlich R, Mognol GP, Robbs BK, Viola JP, Campa A, Amarante-Mendes GR. Melatonin protects CD4+ T cells from activation-induced cell death by blocking NFAT-mediated CD95 ligand upregulation[J]. J Immunol,2010, 184(7): 3487-3494.

[17] Lissoni P, Barni S, Crispino S, Tancini G, Fraschini F. Endocrine and immune effects of melatonin therapy in metastatic cancer patients[J]. Eur J Cancer Clin Oncol, 1989,25(5): 789-795.

[18] Bartsch H, Bartsch C. Effect of melatonin on experimental tumors under different photoperiods and times of administration[J]. J Neural Transm, 1981, 52(4): 269-279.

[19] Lissoni P, Barni S, Mandalà M, Ardizzoia A, Paolorossi F, Vaghi M, Longarini R, Malugani F, Tancini G. Decreased toxicity and increased efficacy of cancer chemotherapy using the pineal hormone melatonin in metastatic solid tumor patients with poor clinical status[J]. Eur J Cancer, 1999, 35(12): 1688-1692.

[20] Lissoni P, Tisi E, Barni S, Ardizzoia A, Rovelli F, Rescaldani R, Ballabio D, Benenti C, Angeli M, Tancini G, Conti A, Maestroni GJM. Biological and clinical results of aneuroimmunotherapy with interleukin-2 and the pineal hormone melatonin as a first line treatment in advanced non-small cell lung cancer[J]. Br J Cancer,1992, 66(1): 155-158.

[21] Lissoni P, Messina G, Rovelli F, Brivio F, Di Fede G. Dose-dependency of antitumor effects of the pineal hormone melatonin in untreatable metastatic solid tumor patients[J]. Int J Immunol Immunobiol,2018,1(1): $1-3$.

[22] Lissoni P, Rovelli F, Brivio F, Messina G, Lissoni A, Pensato S, Di Fede G. Five year-survival with high-dose melatonin and other antitumor pineal hormones in advanced cancer patients eligible of the only palliative therapy[J]. Res J Oncol,2018, 2(1): 1-7.

[23] Nordlund JJ, Lerner AB. The effects of melatonin on skin color and on the release of pituitary hormones[J]. J Clin Endocrinol Metab,1977, 45(4): 768-774. 